\title{
SIMULATIONS OF CRITICAL PROPERTIES OF CLASSICAL SPIN MODELS
}

\author{
P. Kozłowski, P. Pawlicki, G. Kamieniarz
}
Computational Physics Division Institute of Physics, A. Mickiewicz University, ul. Umultowska 85, PL 61-624 Poznań, Poland
e-mail: gjk@pearl.amu.edu.pl

\begin{abstract}
Two methods of simulations are described: an exact one - transfer matrix technique and a statistical one - Monte Carlo method. Both of them are applied to investigate critical properties of classical spin models. To do this we also exploit finite size scaling and the critical point ratio of the square of the second moment of the order parameter to its fourth moment. General definition of a classical spin model as well as particular definitions of models are presented. Results of both methods are in good agreement and, moreover, they are consistent with numerical results provided by literature.
\end{abstract}

\section{$1 \quad$ Classical spin models}

A spin is a quantity hard to understand on the grounds of classical physics. A notion of a spin comes from experimental results and as a physical quantity it is described by a spin operator in the formalism of quantum mechanics. As yet only in this formalism a definition of a spin is reasonable.

Thus, when we talk about spin in the context of classical models we do not mean a quantum spin operator. A name "spin" is used to call a set of numbers labeling states of an atom or a molecule, or in general, a site of a lattice. The name comes from the fact that these numbers are eigenvalues of the $z$ component of a real quantum spin operator. So, defining a quantum model (e.g. of a solid state) we use operators $\hat{\vec{S}}_{i}$ located at each site $i$ of a lattice, but when we define a classical spin model we use variables $S_{i}$ located at each site $i$ of a lattice, where $S_{i}$ is an eigenvalue of a $z$ component of an operator $\hat{\vec{S}}_{i}$.

Classical spin models may be sometimes regarded as approximation of quantum spin models, but not always. Sometimes a spin variable is used to write 
down a Hamiltonian of a model only because of mathematical convenience. In general there are two reasons for which classical spin models are interesting:

- They are usually easier to treat than quantum models and may be used as approximations of the latter, (e.g. Ising model)

- There is a broad spectrum of problems where classical formalism (i.e. classical models) works very well and where quantum models do not introduce any qualitative changes to physics of the problem.

We focused our investigations on the following classical models defined for the nearest neighbours interaction case:

the Ising model - the simplest and the best known one, but still not exactly solved in three dimensions

$$
\begin{gathered}
\beta \mathcal{H}=-\sum_{<i, j>} K_{1} S_{i} S_{j}-h \sum_{i=1}^{N} S_{i} \\
S_{i}= \pm 1
\end{gathered}
$$

$\mathrm{Si}$ is called the Ising variable

the Ashkin-Teller (AT) model - one of the generalisations of the Ising model originally defined as a model of a four component alloy [1], The Hamiltonian of this model may be written using two Ising variables ( $\mathrm{S}$ and $\sigma$ ) located at each site of a lattice [2]:

$$
\begin{gathered}
\beta \mathcal{H}=-\sum_{<i, j>}\left(K_{1} S_{i} S_{j}+K_{2} \sigma_{i} \sigma_{j}+K_{4} S_{i} \sigma_{i} S_{j} \sigma_{j}\right)-h \sum_{i=1}^{N} S_{i} \sigma_{i} \\
S_{i}= \pm 1 ; \quad \sigma_{i}= \pm 1
\end{gathered}
$$

the Extended Ashkin-Teller (EAT) model - an extended version of the AT model proposed by Pawlicki [3]

$$
\begin{gathered}
\beta \mathcal{H}=-\sum_{<i, j>}\left\{K_{1} S_{i} S_{j}+K_{2} \sigma_{i} \sigma_{j}+K_{4} S_{i} \sigma_{i} S_{j} \sigma_{j}+K\left(S_{i}+S_{j}\right)\left(\sigma_{i} \sigma_{j}+1\right)\right\}-h \sum_{i=1}^{N} S_{i} \sigma_{i} \\
S_{i}= \pm 1 ; \quad \sigma_{i}= \pm 1
\end{gathered}
$$


the Blume-Capel (BC) model - proposed independently by Blume [4] and Capel [5].

$$
\begin{gathered}
\beta \mathcal{H}=-K \sum_{<i, j>} S_{i} S_{j}+\Delta \sum_{i} S_{i}^{2}-h \sum_{i=1}^{N} S_{i} \\
S_{i}= \pm 1,0
\end{gathered}
$$

the $q=3$ Potts model - One of $q$-state Potts models. It may be also defined by puting $\sigma_{i}= \pm 1,0$. The model defined in such a way will fit to our definition of a classical spin model.

$$
\begin{array}{r}
\beta \mathcal{H}=-K \sum_{<i, j>} \delta\left(\sigma_{i}, \sigma_{j}\right)-h \sum_{i} \delta\left(0, \sigma_{i}\right), \\
\sigma_{i}=0,1,2 \quad \delta-\text { Kronecker delta }
\end{array}
$$

Everywhere in the Hamiltonians given above $\beta$ stands for inverse temperature $\frac{1}{k_{B} T}$, (where $k_{B}$ is Boltzman constant), $h$ stands for reduced magnetic field and the parameters $K, K_{1}, K_{2}$ and $\Delta$ stand for various coupling constants.

All these models are especially interesting as models of phase transitions in solids.

\section{The critical point and scaling relations}

Two sorts of phase transitions are generally distinguished:

first order phase transitions - the name comes from the fact that at least one of the derivatives of free energy is discontinous at the phase border

continuous phase transitions - First order derivatives of free energy are continuous. Usually the second or higher order derivatives of free energy are discontinuous at the phase border.

A critical point is a point in a phase diagram at which a continuous phase transition takes place. A critical line is a line consisting of critical points. A typical phase diagram of a pure substance is presented in Fig. 1.

A second order phase transition is usually a transition between the ordered and disordered phases. For description of such a transition the so called order parameter is used. This is a quantity which is equal to zero in disordered phase and is different from zero in ordered phase. In magnetic systems, for instance, where 


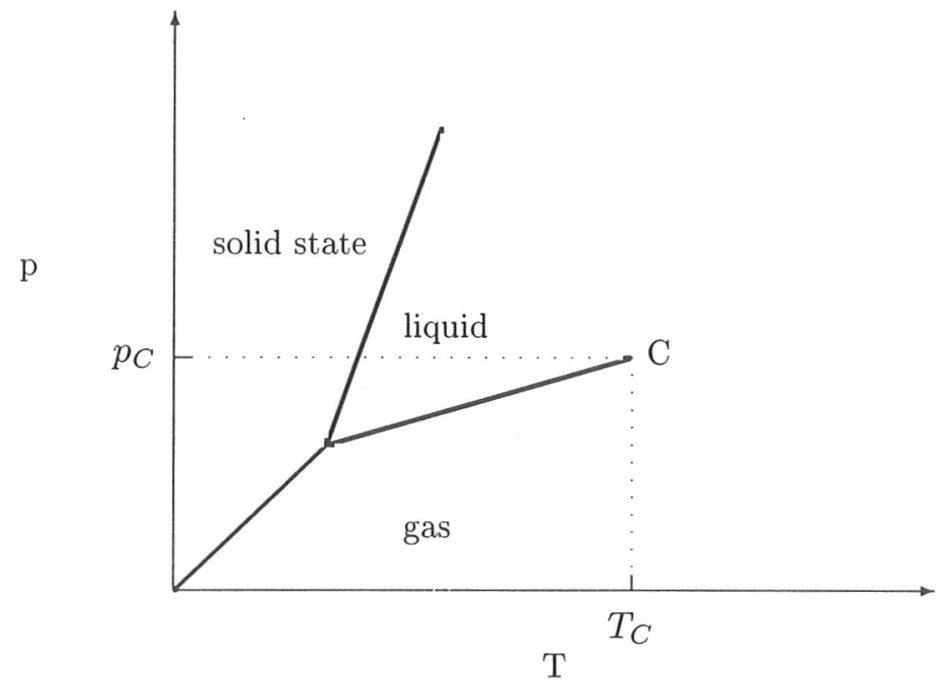

Figure 1: A schematic phase diagram of pure substance. Solid lines are lines of first order phase transition. Point $\mathrm{C}$ is the critical point.

transition between para- and ferromagnetic phases is continuous, magnetization of a lettice is a good order parameter.

In the very close vicinity of the critical point many physical quanities may be scaled with respect to reduced temperature $t=\frac{T-T_{c}}{T_{c}}$ (where $T$ is temperature and $T_{c}$ is temperature at the critical point (critical temperature)), and other scaling fields i.e. quantities defined in such a way that at the critical point all of them are equal to zero. Thus a magnitude of scaling fields is a measure of a distance in the parameter space from the critical point.

It is sufficient to find scaling relations only for free energy since other thermodynamical potentials can be easily calculated therefrom. From experimental results and renormalisation group arguments the following scaling formula [6] for free energy may be written:

$$
f_{s}\left(g_{1}, g_{2}, \ldots, g_{n}\right) \propto l^{-d} f_{s}\left(l^{y_{1}} g_{1}, l^{y_{2}} g_{2}, \ldots, l^{y_{n}} g_{n}\right)
$$

where $\quad(i=1, \ldots, n)$ are scaling fields, $y_{i}(i=1, \ldots, n)$ are the so called critical exponents, $d$ is a dimension of a system, $l$ - rescaling parameter and $f_{s}$ is a singular part of free energy density $f$ defined as follows:

$$
f \equiv \lim _{N \rightarrow \infty} \frac{\mathcal{F}}{N}
$$


Experimental results and some theoretical assumptions [6] allow us to define also other critical exponents:

$$
\begin{aligned}
C_{h} & \propto|t|^{-\alpha} \\
M & \propto|t|^{\beta} \\
\chi_{T} & \propto|t|^{-\gamma} \\
\xi & \propto|t|^{-\nu},
\end{aligned}
$$

where $C_{h}$ - specific heat, $M$ - magnetization, $\chi_{T}$ - magnetic susceptibility, $\xi$ correlation length. Exponents $\alpha, \beta, \gamma, \nu$ are called critical point exponents and are universal, i.e. the same for a given class of models. They are also related to exponents $y_{i}$ from Eq. 6. Because phase transitions take place only in infinite systems we may introduce another scaling field $\frac{1}{L}$, where $L$ is a linear size of a system. Taking $l=L$ we may scale free energy with respect to the linear size of a system:

$$
f_{s}\left(g_{1}, g_{2}, \ldots, g_{n}, L^{-1}\right) \propto L^{-d} f_{s}\left(L^{y_{1}} g_{1}, L^{y_{2}} g_{2}, \ldots, L^{y_{n}} g_{n}, 1\right) .
$$

This relation is very useful because for $g_{i}=0(i=1, \ldots, n)$ it enables extrapolations of the finite-size results to thermodynamical limit.

Scaling relations are usualy exploited to find critical points or critical exponents of a model. In practice, however, not free energy, but other, more convenient quantities are scaled. One of them ( which we usually apply) is an inverse of the Binder cumulant:

$$
Q_{L}=\frac{<M^{2}>_{L}^{2}}{<M^{4}>_{L}}
$$

where $M$ is an order parameter, < .. > stands for thermal average and the index $L$ indicates a linear size of a system. At the critical point the quantity $Q_{\infty}=\lim _{L \rightarrow \infty} Q_{L}$ is universal. Exploiting the relations:

$$
<M^{2}>=\frac{\partial^{2} F}{\partial h^{2}}, \quad<M^{4}>=\frac{\partial^{4} F}{\partial h^{4}}+3\left(\frac{\partial^{2} F}{\partial h^{2}}\right)^{2}
$$

and the scaling the formula (12) we can derive [7] a scaling relation for $Q_{L}$ with respect to the scaling field $\frac{1}{L}$ and expand it with respect to $t$ :

$$
Q_{L}(t)=Q_{L}(0)+\left.\frac{\partial Q_{L}}{\partial t}\right|_{t=0} t+\ldots .
$$

For the Ising universality class [8]: 


$$
\begin{array}{r}
Q_{L}(0)=Q_{\infty}+\beta_{1} L^{2-2 y_{h}}+\left(\beta_{2}+\beta_{3} \ln L\right) L^{1-2 y_{h}}+\beta_{4} L^{4-4 y_{h}} \\
+\beta_{5} L^{-2 y_{h}}+\left(\beta_{6}+\beta_{7} \ln L\right) L^{3-4 y_{h}}+\ldots,
\end{array}
$$

where $y_{h}$ is a magnetic exponent and $\beta_{i}(i=1, \ldots)$ are amplitudes independent of $t$ and $L$. The corresponding formula for $\left.\frac{\partial Q_{L}}{\partial t}\right|_{t=0}$ is more complicated [7] and depends on many terms proportional to $L$ in powers larger than those in the formula (16) for $Q_{L}(0)$.

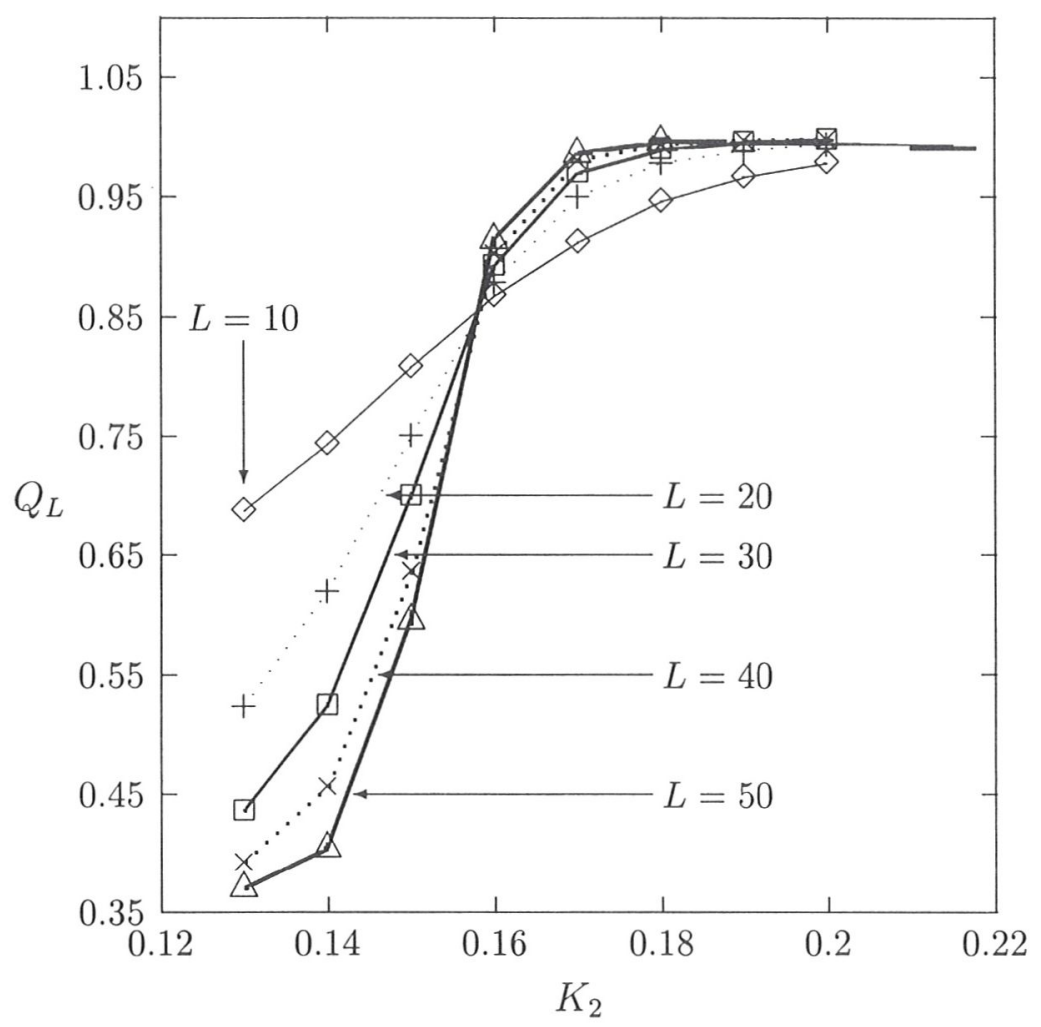

Figure 2: Cumulant ratio $Q_{L}$ for EAT model measured for $K_{4}=0.36, K=0.03$ along the $K_{2}$ axis. Critical value of the parameter $K_{2}$ is 0.158 and the system sizes are defined by the labels.

Now we will focus on the method of finding the exact position of the critical point. We take all the scaling fields except $t$ and $L^{-1}$ equal to zero. Then we compute $Q_{L}(t)$ (using transfer matrix (TM) or Monte Carlo (MC) method) for various $L$ at a starting point. It is the point on the phase diagram which lies 
close to the supposed position of the critical line. Then putting values of $Q_{L}(t)$ into formula (15) we obtain a set of equations for amplitudes $\beta_{i}$ and for $t$. The larger the $L$ is the more terms in expansion (16) for $Q_{L}(\mathrm{t})$ can be considered, and the more accurate our results are. Amplitudes $\beta_{i}$ can be calculated for a known $t$ at one point and can be used for finding other points because they are quite stable within one model (however, they are not universal). It is possible for models where at least one critical point is known exactly (e.g. the Ising point in the AT model).

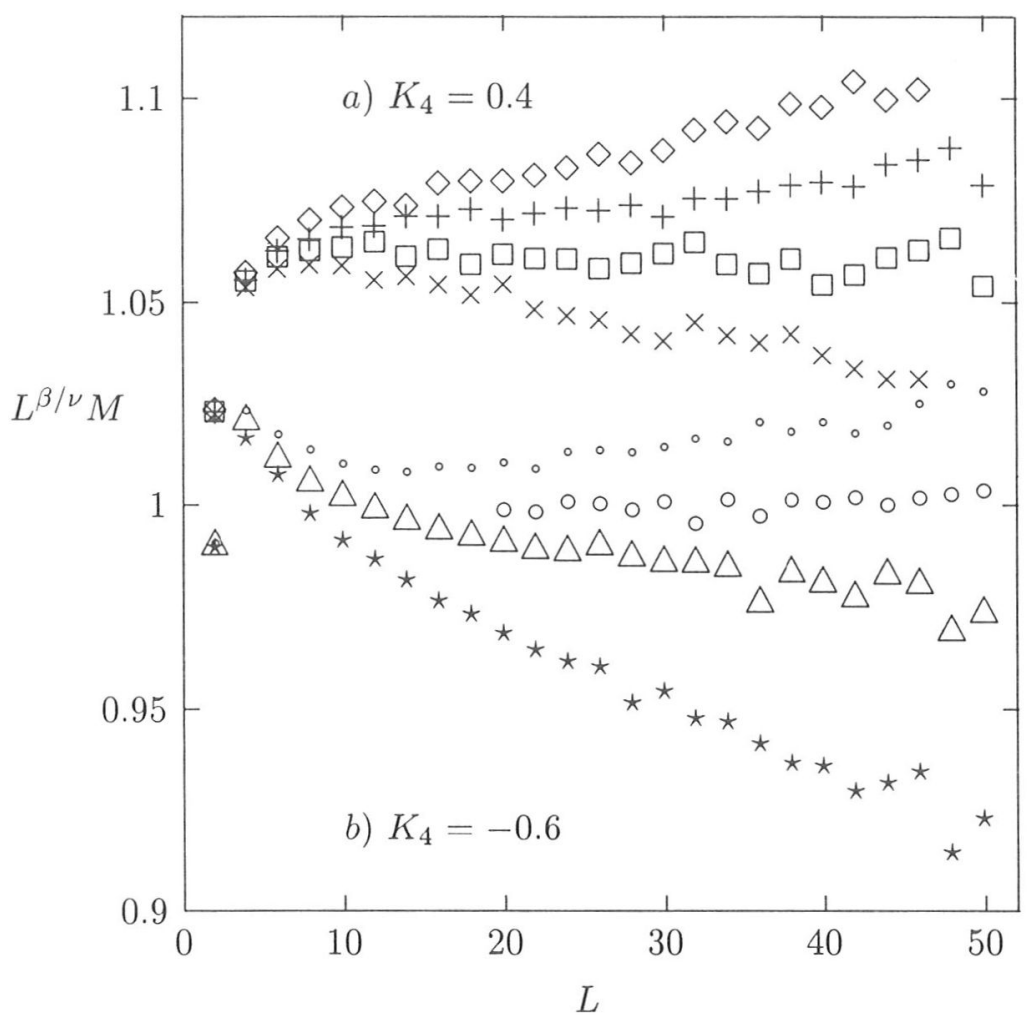

Figure 3: Size-scaled values of the order parameter $L^{\beta / \mu} M$ (for the EAT model) measured for different values of linear size $L$, assuming the values of the Ising critical exponents. The parameters are:

a) $K=0.04, K_{2}=[\times] 0.102,[\square] 0.103,[+] 0.104,[\diamond] 0.105$

b) $K=0.1, K_{2}=[\star] 0.275,[\triangle] 0.273,[\bigcirc] 0.272,[\circ] 0.271$.

Solving the above mentioned set of equations we obtain a value of the scaling field $t$. Since $t$ is a measure of a distance from the critical point, knowing the coordinates of the starting point we calculate coordinates (in the parameter space) of the critical point. This method is very accurate and works even for 
very small systems $[7,8,9,10]$, provided that the accurate finite-size estimates are found.

Because $Q_{L}$ at the critical point very quickly tends towards the universal value $Q_{\infty}$ with increasing $L$, having results for large $L$ we can assume that at the critical point $Q_{L}=Q_{\infty}$. This is of course an approximation which becomes exact as $L$ tends to infinity. Thus, if we compute numericaly $Q_{L}$ for large $L$ (using MC method) for various values of parameters we may present them as curves plotted against certain parameters (e.g. temperature $t$ ). For another $L$ we have another curve and so on. Since $Q_{\infty}$ does not depend on $L$, the curves should intersect for the critical values of the parameters (Fig. 2). In fact, for small $L$, there is not one point of intersection but a series of points, which go to one limiting point as $L$ tends to infinity. This limiting point is the critical point we are looking for. The method based on the Binder cumulant has been succesfuly applied to a variety of models $[11,12,13,14,15,16,17,18,19,20]$.

If we consider large systems we can neglect all terms in the scaling formula except one. Then the scaling relation at the critical point for the order parameter $M$ may be written in the form:

$$
M\left(T=T_{c}\right) \propto L^{-\beta / \nu} .
$$

This relation suggests that $M L^{\beta / \nu}$ should be a straight line independent of $L$, if we have choosen the proper values for $T_{c}$ and the critical exponents. Examples of such analyses are shown in Fig. 3 where we plotted the $L$-dependence of $M L^{\beta / \nu}$ for the EAT model for several values of $K_{2}$ in the ferro and antiferromagnetic $\left(K_{4}<0\right)$ regions. This method enables a determination of the critical value of a parameter $K_{2}$ with an accuracy of 0.001 [21].

\section{The transfer matrix method}

The TM method is an exact method exploited by us to compute the ratio $Q_{L}$. It can be applied to a broad class of models written in the form:

$$
\overline{\mathcal{H}}\left(\vec{\Sigma}_{1}, \vec{\Sigma}_{2}, \ldots, \vec{\Sigma}_{L}\right)=\overline{\mathcal{H}}\left(\vec{\Sigma}_{1}, \vec{\Sigma}_{2}\right)+\overline{\mathcal{H}}\left(\vec{\Sigma}_{2}, \vec{\Sigma}_{3}\right)+\ldots+\overline{\mathcal{H}}\left(\vec{\Sigma}_{L}, \vec{\Sigma}_{1}\right)
$$

Vectors $\vec{\Sigma}_{i}(i=1, \ldots, L)$ stand for spin variables from the $\mathrm{i}$-th block of the lattice. In two dimensions and for nearest neighbour interactions, a block is equivalent to a column (Fig. 4).

Then we can express a partition function as follows:

$$
\mathcal{Z}=\operatorname{Tr} e^{-\overline{\mathcal{H}}}=\sum_{\vec{\Sigma}_{1}, \ldots, \vec{\Sigma}_{L}} e^{-\overline{\mathcal{H}}\left(\vec{\Sigma}_{1}, \vec{\Sigma}_{2}\right)} e^{-\overline{\mathcal{H}}\left(\vec{\Sigma}_{2}, \vec{\Sigma}_{3}\right)} \ldots e^{-\overline{\mathcal{H}}\left(\vec{\Sigma}_{L}, \vec{\Sigma}_{1}\right)}=\sum_{\vec{\Sigma}_{1}} \mathbf{T}^{L}\left(\vec{\Sigma}_{1}, \vec{\Sigma}_{1}\right)
$$




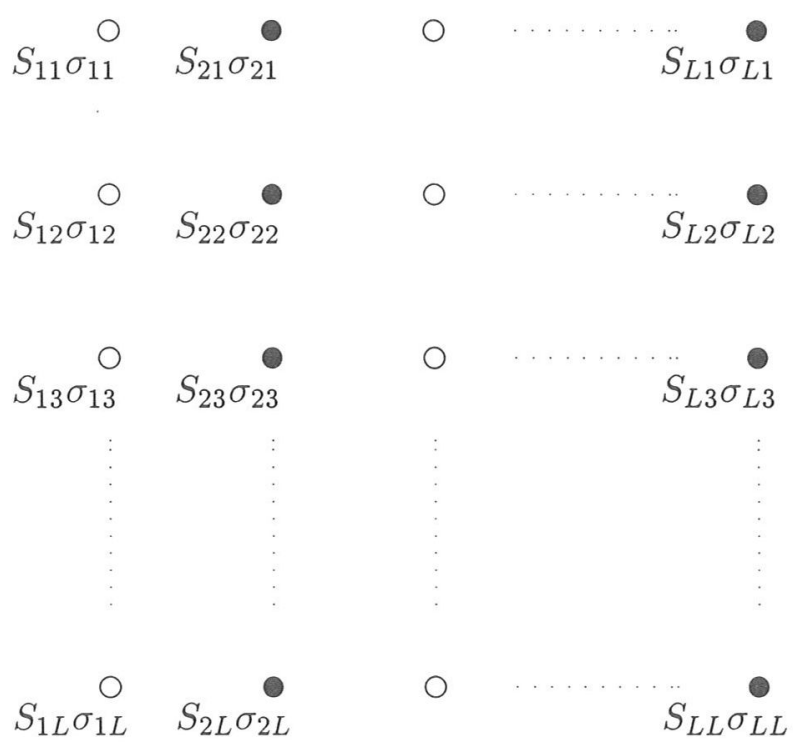

Figure 4: Blocks of Ising variables for the AT model (for nn interactions) on square lattice. One column is equivalent to one block.

where $\mathbf{T}\left(\vec{\Sigma}_{i}, \vec{\Sigma}_{j}\right)=e^{-\overline{\mathcal{H}}\left(\vec{\Sigma}_{i}, \vec{\Sigma}_{j}\right)}$ is called a transfer matrix. This can be split into the product $\mathbf{T}=\mathbf{T}_{h} \mathbf{T}_{v}$ of a diagonal matrix $\mathbf{T}_{v}$ and a non-diagonal matrix $\mathbf{T}_{h}$ containing the intra- and the inter-column interactions, respectively. For the isotropic AT model $\left(K_{l}=K_{2}\right)$ they are defined as follows:

$$
\begin{array}{r}
\mathbf{T}_{v}\left(\vec{\Sigma}_{k}, \vec{\Sigma}_{l}\right)=\delta_{\vec{\Sigma}_{k}, \vec{\Sigma}_{l}} \exp \left(\sum _ { i = 1 } ^ { N } \left(K_{2} S_{k, i} S_{k, i+1}+K_{2} \sigma_{k, i} \sigma_{k, i+1}+\right.\right. \\
\left.\left.+K_{4} S_{k, i} \sigma_{k, i} S_{k, i+1} \sigma_{k, i+1}+h S_{k, i} \sigma_{k, i}\right)\right) \\
\mathbf{T}_{h}\left(\vec{\Sigma}_{k}, \vec{\Sigma}_{l}\right)=\exp \left(\sum_{i=1}^{N}\left(K_{2} S_{k, i} S_{l, i}+K_{2} \sigma_{k, i} \sigma_{l, i}+K_{4} S_{k, i} \sigma_{k, i} S_{l, i} \sigma_{l, i}\right)\right) .
\end{array}
$$

The symbols in eqs (20) and (21) are the same as in eq. (2). The matrix $\mathbf{T}_{h}$ can be expressed as a product of sparse matrices what facilitates the numerical calculations.

The averages in Eq. (13) can be expressed in terms of the corresponding coefficients $Z_{k}$ [8] in the expansion of the field dependent partition function 


$$
\left(Z(h)=\sum_{k=0}^{\infty} Z_{k} \frac{h^{k}}{k !}\right) .
$$

$$
<M^{k}>_{h=0}=\frac{Z_{k}}{Z_{0}}
$$

Only the matrix $\mathbf{T}_{v}$ depends on $h$ and it can be expanded as follows:

$$
\mathbf{T}_{v}(h)=\sum_{k=0}^{\infty} \frac{h^{k}}{k !} \mathbf{T}_{v}^{(k)}(0), \quad \mathbf{T}_{v}^{(k)}(0)=\mathbf{M}^{k} \mathbf{T}_{v}(0)
$$

where $\mathbf{M}$ is a diagonal matrix representing the order parameter; for the AT model it may have the form:

$$
\mathrm{M}\left(\vec{\Sigma}_{i}, \vec{\Sigma}_{j}\right)=\left(\sum_{k=0}^{L} S_{i, k} \sigma_{i, k}\right) \delta_{\vec{\Sigma}_{i}, \vec{\Sigma}_{j}}
$$

The coefficients $Z_{k}$ can then be calculated from Eq. (19) (which may have the form of a sum over all base vectors of one block: $\left.\mathcal{Z}=\sum_{i}\left\langle i\left|\left(\mathbf{T}_{h} \mathbf{T}_{v}\right)^{L}\right| i\right\rangle\right)$ by multiplying the base vectors by matrices $\mathbf{T}_{v}$ and $\mathbf{T}_{h}$ in such a manner that the terms in the same power of $h$ are kept separately [8]. Additionaly we exploit the symmetry of the model, i.e. we consider only non-equivalent states of the block. Such a method facilitates numerical calculations and enables, e.g. in case of the Ising model, exact calculation of $Q_{L}$ ratio for two dimensional systems up to size $20 \times 20$ in a reasonable time. For more complicated models the maximum size of the system for which calculations can be performed is somewhat smaller.

\section{The Monte Carlo method}

The second computational method used by us is the MC method. It enables consideration of bigger systems, but with lower accuracy due to the statistical nature of the method. In our investigation we generate numerically two-dimensional finite size samples of spins for fixed values of the model parameters. Periodic boundary conditions are imposed and thermalization of the initial configurations (relaxation of the system towards thermal equilibrium) is applied. For such a sample all equilibrium physical quantities as well as their statistical errors may be obtained by a direct calculation.

One possible strategy for sampling the configurations of a system is to choose states completely at random. However, there is a serious problem with this approach. If the states which make negligible contributions to such quantities as partition function are taken with the same weight, they give a great unphysical contribution to the variance of the internal energy which leads to serious errors at low temperatures. We can alleviate this problem by the so called importance sampling which consists in arranging the way of sampling so that we spend 
as much time as possible looking at those configurations which make a large contribution to the quantities we are trying to calculate.

We sample the Gibbs distribution using the Metropolis algorithm [22]. We start with some initial configuration $\alpha$ of spins and a new configuration $\alpha^{\prime}$ of the system is generated from a by some method. For example, one might try reversing the direction of a single spin. This spin may be selected at random, or each of the spins in the sample may be reversed in turn (which is the case here). We reverse the spin or do not do it according to some initially chosen transition probability and when each spin of the sample has been visited once (on the average or consecutively) we carried out one Monte Carlo step (MCS) per spin. Either of these procedures ensures that the accessibility criterion is satisfied, which states that it must be possible to evolve the system from a given starting point to any of its other configurations by applying the evolution rule a sufficiently large number of times.

In order to decide whether to accept a single spin-flip or not, we compare energies of the new and old configurations. If the energy change is negative, then the new configuration is automatically accepted; if, however, it is positive, the new configuration is accepted with a probability $e^{-\beta\left(E_{\alpha^{\prime}}-E_{\alpha}\right)}$. For the Metropolis algorithm the microreversibility assumption is satisfied which means that we have:

$$
p_{\alpha} P\left(\alpha \rightarrow \alpha^{\prime}\right)=p_{\alpha^{\prime}} P\left(\alpha^{\prime} \rightarrow \alpha\right),
$$

where $p_{\alpha}$ denotes a probability of the configuration $\alpha$, while $P\left(\alpha \rightarrow \alpha^{\prime}\right)$ stands for a probability of the transition from $\alpha$ to $\alpha^{\prime}$ configuration. Physically it means that both configurations are in equilibrium and none of them arises at the expense of the other. In such a way we generate a Markov process which gives us an insight into a dynamical evolution of the initial configuration towards an equilibrium. Using this method, we generate samples which allow us to calculate physical quantities in a direct way [21].

\section{$5 \quad$ Results}

In this section we present the results and compare them with those available in literature. For the isotropic AT model on a square lattice we calculated the position of the Ising like critical lines exploiting TM technique (Fig. 5).

In the phase diagram we can distinguish the four phases:

B - Baxter phase $(<S\rangle \neq 0,<S \sigma\rangle \neq 0)$

$\mathbf{P}$ - paramagnetic phase $(\langle S\rangle=0,\langle S \sigma\rangle=0)$

F - ferromagnetic phase $(\langle S\rangle=0,\langle S \sigma\rangle \neq 0)$ 


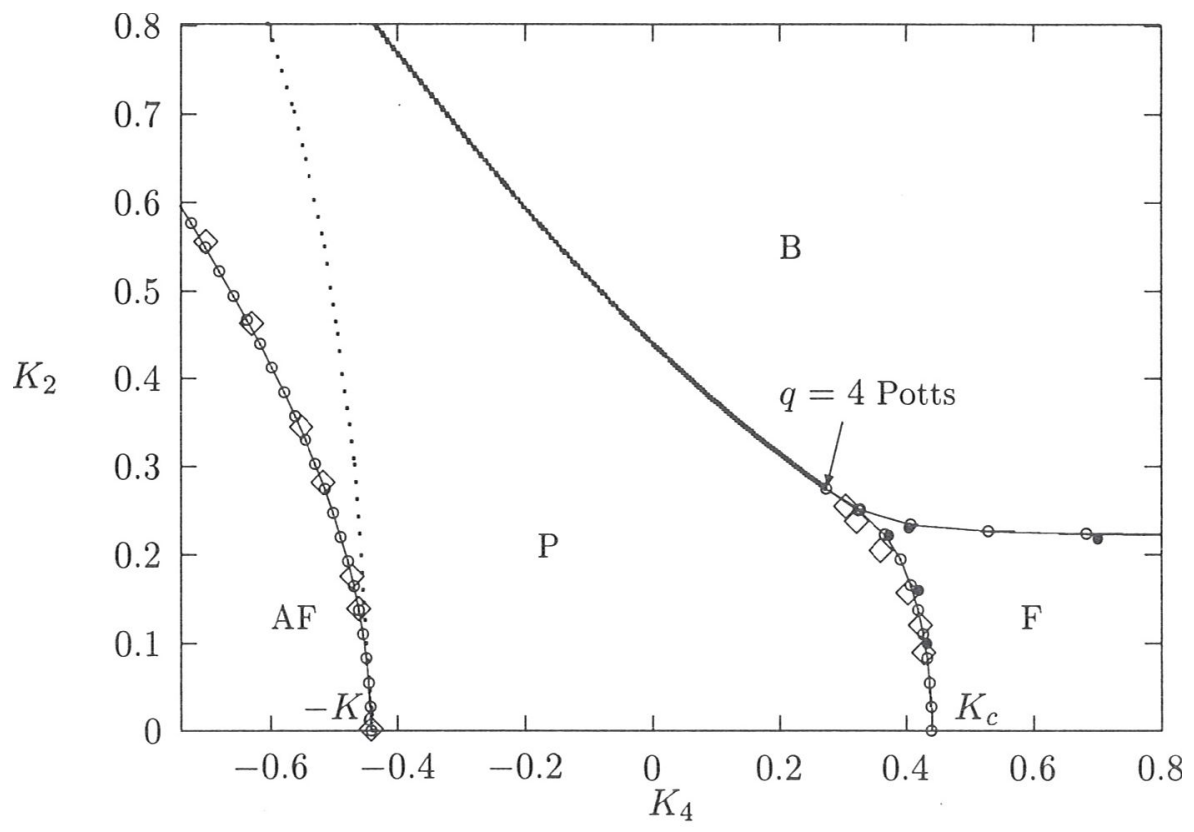

Figure 5: The phase diagram of the AT model in two dimensions. The solid bold line represents the exactly known critical line, which terminates at the 4state Potts point. Empty circles with continuous lines describe our results. The solid circles display MCRG results, the dotted line is drawn after Baxter and diamonds are the transfer matrix results combined with conformal invariance.

AF - antiferromagnetic phase $(<S>=0,\langle S \sigma>\neq 0$ on sublattice i.e. $S \sigma$ ordered in an antiferromagnetic way),

The first two order parameters $\langle S\rangle$ and $\langle S \sigma\rangle$ are different. The third order parameter $\langle\sigma\rangle$ is always equal to $\langle S\rangle$ because we consider the isotropic $\left(K_{1}=K_{2}\right)$ version of the model.

The curve plotted by the bold line represents the part of the phase diagram found exactly by Baxter [23]. It separates the Baxter phase B from the paramagnetic phase $P$.

In the ferromagnetic region $K_{4}>0$ we have only calculated the curve joining the 4-state Potts point to the pure Ising point $K_{c}$ at $K_{2}=0$. The second branch follows from the corresponding duality relation [24, 23]. The boundary between $\mathrm{AF}$ and $\mathrm{P}$ phases is plotted in Fig. 5 by the dotted line given approximately by Baxter [23] and in the ferromagnetic region we also include the MCRG results [25] marked by filled circles. 


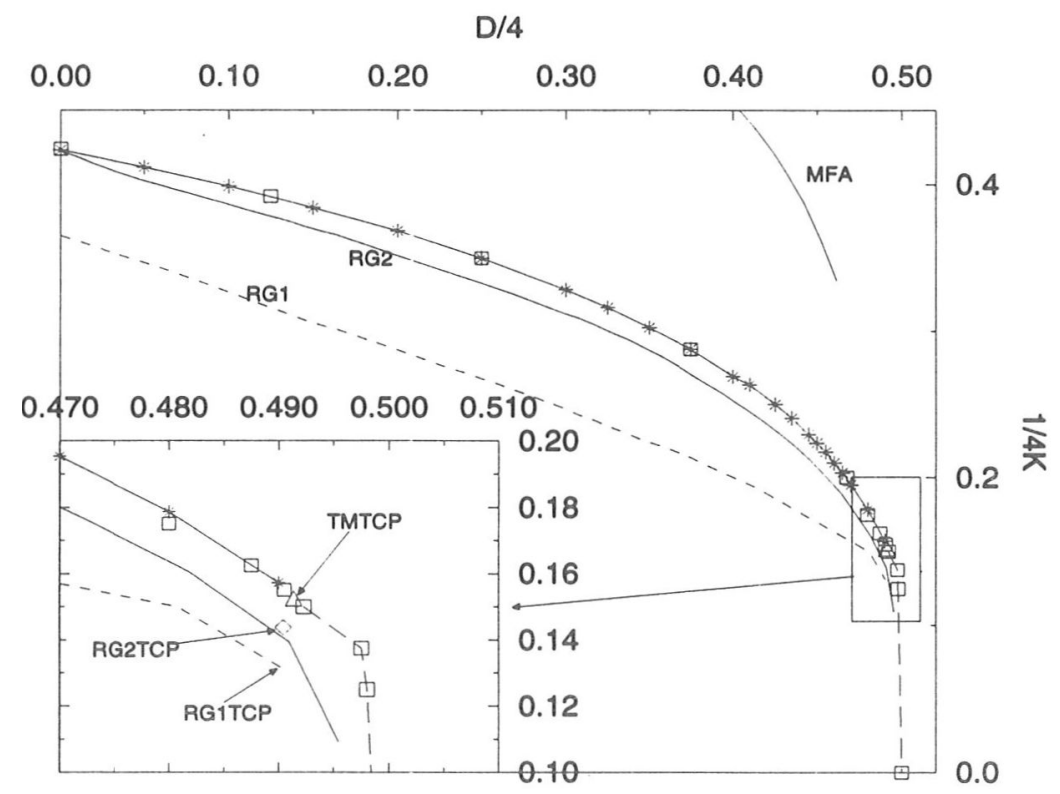

Figure 6: The phase diagram of the Blume-Capel model $(D=\Delta / K)$. Our results are drawn with stars connected by lines. RG1 and RG2 are lines of continuous transition predicted by two different RG approaches. Earlier results obtained in PM scheme are drawn with squares and a long dashed line connects points which belong to the first order transiton line.

As can be seen (Fig. 5), our results are in good agreement with the MCRG [25] approach, but they are quite different from the Baxter's predictions [23] in the antiferromagnetic region. For the boundary between AF and $\mathrm{P}$ phases, our results coincide with those obtained by Mazzeo et al. [26]. These authors actually investigated the six vertex model with the transfer matrix technique in combination with conformal invariance arguments; their results can be mapped onto the results for the P-phase boundaries and they are shown in Fig. 5. The numerical uncertainties do not exceed the size of the symbol.

In Fig. 6 the results obtained for the BC model are presented. As in the case of the AT model we calculated with high accuracy the position of the Ising like critical line by recourse of the $Q$-ratio technique. This line ends at the tricritical point (TCP) which is located using the phenomenological renormalization scheme (PR) [27] and is denoted in Fig. 6 by TMTCP) and two different real-space renormalisation group approaches [28] (denoted as RG1TCP and RG2TCP). At the TCP the critical line meets the first order transition line (long 
dashed line). We did not calculate the TCP because its position is known with quite a high accuracy from PR [27] $\left(1 / K_{t}=0.610(5), D_{t}=1.965(5)\right)$ and MC results obtained by Wilding at al [29] $\left(1 / K_{t}=0.608(1), D_{t}=1.9665(3)\right)-$ not shown in Fig. 6. These results coincide within the error bars and we did not see a need to estimate them once again, especially, because this point belongs to a different universality class. Determination of its positions requires more effort than in case of an Ising like transition. Our results (stars connected by solid line in Fig. 6) are in good agreement with PR results (squares in Fig. 6) and demonstrate the systematic deviations of those obtained in the framework of RG approaches [28].

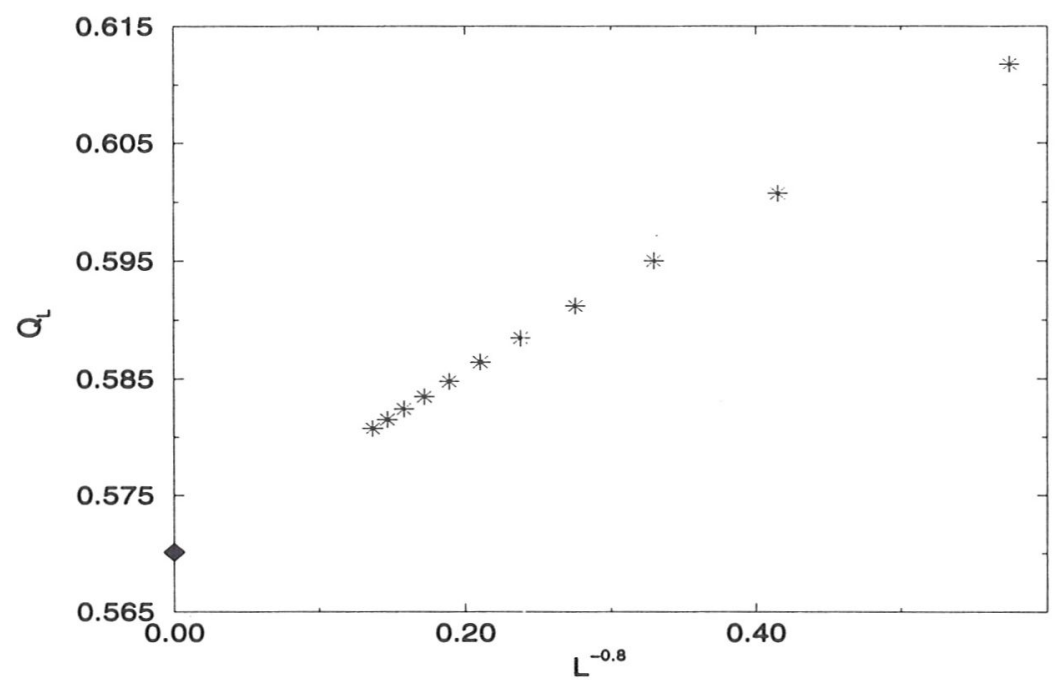

Figure 7: The size dependence of the $Q_{L}$ ratio at the critical point of the $q=3$ Potts model. The stars denote $Q_{L}$ and the filled diamond shows an extrapolated value.

Fig. 7 presents the $Q_{L}$ ratio plotted against $L^{-0.8}$ at the critical point of the $q=3$ Potts model on a square lattice $L \times L$. The linear character of this dependence confirms the theoretical scaling predictions [30]. Results from Fig. 7 enable an estimation of the universal critical point ratio at $Q_{\infty}=0.5701 \pm 0.0001$, which has not been known so far.

The phase diagram of the EAT model obtained by MC method is presented in Fig. 8. Open circles and stars stand for our MC and TM results, respectively. Error bars do not exceed the size of the symbols. The plane $K=0$ in Fig. 8 is the phase diagram of the isotropic AT model. It is easy to perceive that the MC and TM results coincide within the error bars. In the framework of MC 
simulations (Fig. 8) two surfaces of Ising like transitions have been calculated and the existence of the tricritical points has been ruled out.

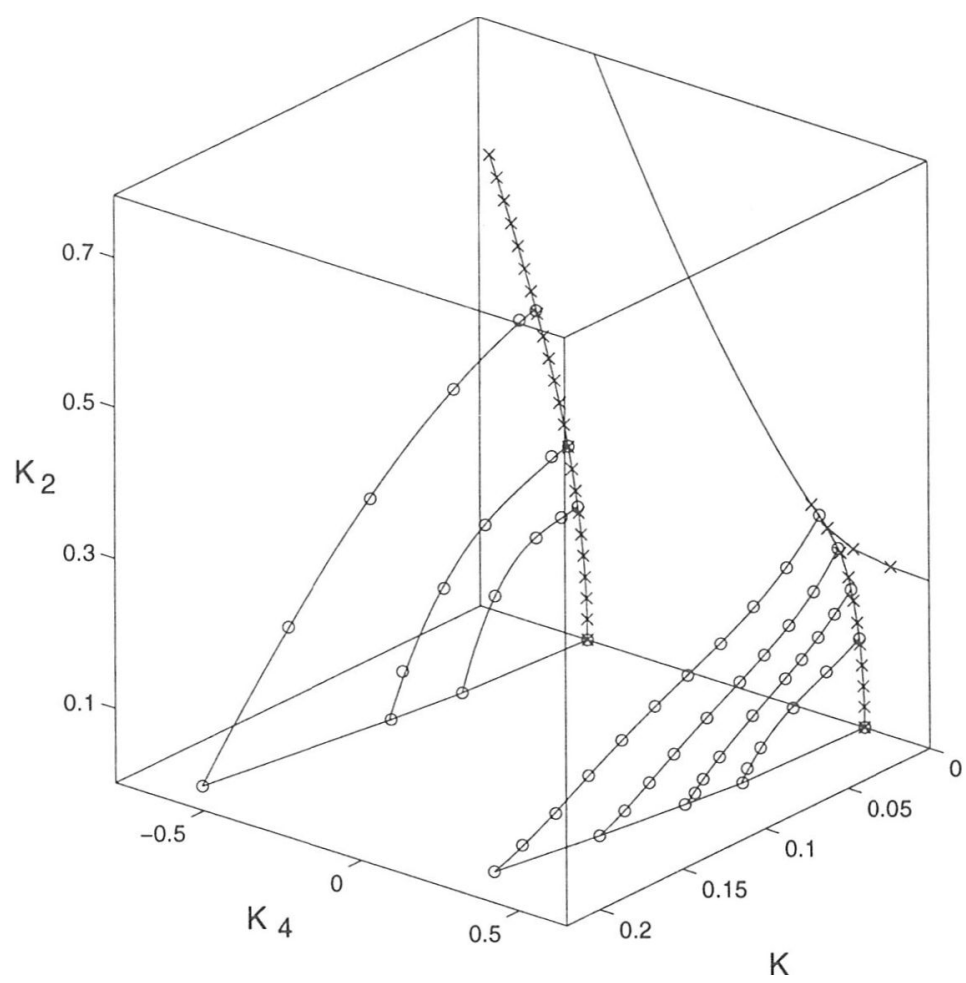

Figure 8: The critical surfaces in the space of parameters. Circles denote the results obtained by $\mathrm{MC}$ method, stars denote the values from the transfer matrix technique. The line without any symbol represents the exact Baxter curve. Other lines are drawn to guide eyes.

\section{$6 \quad$ Acknowledgments}

We thank Dr. E. Carlon, Dr. J.R. Heringa and Prof. J. Rogiers for discussions as well as Z. Brudzyński and A. Witek for their help with the graphics. The numerical calculations were carried out in the Supercomputing and Networking Center in Poznań on Cray J-916. The work has been supported in part by the Committee for the Scientific Research via grant 8 Til F 01509. 


\section{References}

[1] J. Ashkin and E. Teller, Phys. Rev. 64 (1943), 178.

[2] C. Fan, Phys. Lett. 39A (1972), 136.

[3] P. Pawlicki, J. Rogiers Physica A 214 (1995), 277.

[4] M. Blum Phys. Rev. 141 (1966), 517.

[5] H. W. Capel, Physica (Utr.) 32 (1966), 966; 33 (1967), 295; 37 (1967), 423.

[6] H. E. Stanley Introduction to Phase Transitions and Critical Phenomena, Clarendon Press, Oxford 1971.

[7] G. Kamieniarz, P. Kozłowski and R. Dekeyser, Phys.Rev. E 55 (1997), 3724.

[8] G. Kamieniarz and H. W. J. Blöte, J. Phys. A 26 (1993), 201.

[9] H. W. J. Blöte and G. Kamieniarz, Physica A 196 (1993), 455.

[10] G. Kamieniarz, P. Kozłowski and R. Dekeyser, Acta Magnetica, XI 1996/1997.

[11] K. Binder, Z. Phys. B 43 (1981), 119.

[12] A. Milchev, D. W. Heermann, and K. Binder, J. Stat. Phys. 44 (1986), 749.

[13] K. Binder and D. P. Landau, Phys. Rev. Lett. 52 (1984), 318.

[14] K. K. Kaski, K. Binder, and J. D. Gunton, Phys. Rev. B 29 (1984), 3996.

[15] M. N. Barber, R. B. Pearson, D. Toussaint and J. L. Richardson, Phys. Rev. B 32 (1985), 1720.

[16] D. P. Landau, J. Magn. Magn. Mater. 31 (1983), 11.

[17] H. W. J. Blöte, E. Luijten and J. R. Herringa, J. Phys. A 28 (1995), 6289.

[18] D. P. Landau and K. Binder, Phys. Rev. B 31 (1985), 5946.

[19] K. Binder, M. Nauenberg, V. Privman and A. P. Young, Phys. Rev. B 31 (1985), 1498.

[20] R. N. Bhatt and A. P. Young; Phys. Rev. Lett. 54 (1985), 924.

[21] G. Kamieniarz, P. Pawlicki, L. Dębski, and H.W.J. Blöte, Acta Magnetica XI 1996/1997.

[22] N. Metropolis, A. Rosenbluth, M. Rosenbluth, A. Teller and E. Teller, J. Chem. Phys. 21 (1953), 1087.

[23] R. J. Baxter, Exactly Solved Models in Statistical Mechanics (Academic, New York, 1982).

[24] R. V. Ditzian, J. R. Banavar, G. S. Grest and L. P. Kadanoff, Phys. Rev. B 22 (1980), 2542.

[25] J. Chahine, J. R. Drugowich de Felicio and N. Caticha, J. Phys. A 22 (1989), 1639.

[26] G. Mazzeo, E. Carlon and H. van Beijeren, Phys. Rev. Lett. 74 (1995), 1391.

[27] P. D. Beale, Phys. Rev. B 33 (1986), 1717.

[28] T. W. Burkhardt, Phys. Rev. B 14 (1967), 1196;

T. W. Burkhardt and H. J. F. Knops, Phys.Rev. B 15 (1977), 1602.

[29] N. B. Wilding and P. Nielaba, Phys. Rev. E 53 (1996), 926.

[30] H. W. J. Blöte and M. P. M. de Nijs, Phys. Rev. B 37 (1988), 1766. 